\title{
Fixed point of Suzuki-Zamfirescu hybrid contractions in partial metric spaces via partial Hausdorff metric
}

\section{Abbas ${ }^{1}$ and Basit Ali ${ }^{1,2^{*}}$}

\section{"Correspondence:}

basit.aa@gmail.com

${ }^{1}$ Department of Mathematics, Lahore University of Management Sciences, Lahore, 54792, Pakistan Full list of author information is

available at the end of the article

\section{空 Springer}

\begin{abstract}
Coincidence point theorems for hybrid pairs of single-valued and multi-valued mappings on an arbitrary non-empty set with values in a partial metric space using a partial Hausdorff metric have been proved. As an application of our main result, the existence and uniqueness of common and bounded solutions of functional equations arising in dynamic programming are discussed.
\end{abstract}

MSC: 47H10; 54H25; 54E50

Keywords: coincidence point; orbitally complete; common fixed point; partial metric space

\section{Introduction and preliminaries}

Fixed point theory plays a fundamental role in solving functional equations [1] arising in several areas of mathematics and other related disciplines as well. The Banach contraction principle is a key principle that made a remarkable progress towards the development of metric fixed point theory. Markin [2] and Nadler [3] proved a multi-valued version of the Banach contraction principle employing the notion of a Hausdorff metric. Afterwards, a number of generalizations (see [4-9]) were obtained using different contractive conditions. The study of hybrid type contractive conditions involving single-valued and multivalued mappings is a valuable addition to the metric fixed point theory and its applications (for details, see [10-14]). Among several generalizations of the Banach contraction principle, Suzuki's work [15, Theorem 2.1] led to a number of results (for details, see [13, 16-21]).

On the other hand, Matthews [22] introduced the concept of a partial metric space as a part of the study of denotational semantics of dataflow networks. He obtained a modified version of the Banach contraction principle, more suitable in this context (see also $[23,24])$. Since then, results obtained in the framework of partial metric spaces have been used to constitute a suitable framework to model the problems related to the theory of computation (see [22, 25-28]). Recently, Aydi et al. [29] initiated the concept of a partial Hausdorff metric and obtained an analogue of Nadler's fixed point theorem [3] in partial metric spaces.

The aim of this paper is to obtain some coincidence point theorems for a hybrid pair of single-valued and multi-valued mappings on an arbitrary non-empty set with values in a partial metric space. Our results extend, unify and generalize several known results in the existing literature (see $[13,15,21,30])$. As an application, we obtain the existence and

() 2013 Abbas and Ali; licensee Springer. This is an Open Access article distributed under the terms of the Creative Commons Attribution License (http://creativecommons.org/licenses/by/2.0), which permits unrestricted use, distribution, and reproduction in any medium, provided the original work is properly cited. 
uniqueness of a common and bounded solution for Suzuki-Zamfirescu class of functional equations under contractive conditions weaker than those given in [1,31-34].

Throughout this work, a mapping $\omega:[0,1) \rightarrow\left(\frac{1}{2}, 1\right]$ is defined by

$$
\omega(r)=\frac{1}{1+r} \quad \text { for all } r \in[0,1) .
$$

In the sequel, the letters $\mathbb{R}, \mathbb{R}^{+}$and $\mathbb{N}$ will denote the set of all real numbers, the set of all non-negative real numbers and the set of all positive integers, respectively. Consistent with $[22,29,35,36]$, the following definitions and results will be needed in the sequel.

Definition 1.1 [22] Let $X$ be any non-empty set. A mapping $p: X \times X \rightarrow \mathbb{R}^{+}$is said to be a partial metric if and only if for all $x, y, z \in X$ the following conditions are satisfied:

(P1) $p(x, x)=p(y, y)=p(x, y)$ if and only if $x=y$;

(P2) $p(x, x) \leq p(x, y)$;

(P3) $p(x, y)=p(y, x)$;

(P4) $p(x, z) \leq p(x, y)+p(y, z)-p(y, y)$.

The pair $(X, p)$ is called a partial metric space. If $p(x, y)=0$, then (P1) and (P2) imply that $x=y$. But the converse does not hold in general. A classical example of a partial metric space is the pair $\left(\mathbb{R}^{+}, p\right)$, where $p: X \times X \rightarrow \mathbb{R}^{+}$is defined as $p(x, y)=\max \{x, y\}$ (see also [37]).

Example 1.2 [22] If $X=\{[a, b]: a, b \in \mathbb{R}, a \leq b\}$, then

$$
p([a, b],[c, d])=\max \{b, d\}-\min \{a, c\}
$$

defines a partial metric $p$ on $X$.

For more interesting examples, we refer to [23, 27, 28, 35, 38, 39]. Each partial metric $p$ on $X$ generates a $T_{0}$ topology $\tau_{p}$ on $X$ which has as a base the family of open balls ( $p$-balls) $\left\{B_{p}(x, \varepsilon): x \in X, \varepsilon>0\right\}$, where

$$
B_{p}(x, \varepsilon)=\{y \in X: p(x, y)<p(x, x)+\varepsilon\}
$$

for all $x \in X$ and $\varepsilon>0$. A sequence $\left\{x_{n}\right\}$ in a partial metric space $(X, p)$ is called convergent to a point $x \in X$ with respect to $\tau_{p}$ if and only if $p(x, x)=\lim _{n \rightarrow \infty} p\left(x, x_{n}\right)$ (for details, see [22]). If $p$ is a partial metric on $X$, then the mapping $p^{S}: X \times X \rightarrow \mathbb{R}^{+}$given by $p^{S}(x, y)=$ $2 p(x, y)-p(x, x)-p(y, y)$ defines a metric on $X$. Furthermore, a sequence $\left\{x_{n}\right\}$ converges in a metric space $\left(X, p^{S}\right)$ to a point $x \in X$ if and only if

$$
p(x, x)=\lim _{n \rightarrow \infty} p\left(x, x_{n}\right)=\lim _{n, m \rightarrow \infty} p\left(x_{n}, x_{m}\right) .
$$

Definition 1.3 [22] Let $(X, p)$ be a partial metric space, then

(a) A sequence $\left\{x_{n}\right\}$ in $X$ is called Cauchy if and only if $\lim _{n, m \rightarrow \infty} p\left(x_{n}, x_{m}\right)$ exists and is finite.

(b) A partial metric space $(X, p)$ is said to be complete if every Cauchy sequence $\left\{x_{n}\right\}$ in $X$ converges with respect to $\tau_{p}$ to a point $x \in X$ such that $p(x, x)=\lim _{n, m \rightarrow \infty} p\left(x_{n}, x_{m}\right)$. 
Lemma A [22,35] Let $(X, p)$ be a partial metric space, then

(c) A sequence $\left\{x_{n}\right\}$ in $X$ is Cauchy in $(X, p)$ if and only if it is Cauchy in $\left(X, p^{S}\right)$.

(d) A partial metric space $(X, p)$ is complete if and only if $\left(X, p^{S}\right)$ is complete.

Consistent with [29], let $C B^{p}(X)$ be the family of all non-empty, closed and bounded subsets of the partial metric space $(X, p)$, induced by the partial metric $p$. Note that closedness is taken from $\left(X, \tau_{p}\right)\left(\tau_{p}\right.$ is the topology induced by $\left.p\right)$ and boundedness is given as follows: $A$ is a bounded subset in $(X, p)$ if there exists an $x_{0} \in X$ and $M \geq 0$ such that for all $a \in A$, we have $a \in B_{p}\left(x_{0}, M\right)$, that is, $p\left(x_{0}, a\right)<p(a, a)+M$. For $A, B \in C B^{p}(X)$ and $x \in X$, define $\delta_{p}: C B^{p}(X) \times C B^{p}(X) \rightarrow[0, \infty)$ and

$$
\begin{aligned}
& p(x, A)=\inf \{p(x, a): a \in A\}, \\
& \delta_{p}(A, B)=\sup \{p(a, B): a \in A\}, \\
& \delta_{p}(B, A)=\sup \{p(b, A): b \in B\}, \\
& H_{p}(A, B)=\max \left\{\delta_{p}(A, B), \delta_{p}(B, A)\right\} .
\end{aligned}
$$

It can be verified that $p(x, A)=0$ implies $p^{S}(x, A)=0$, where $p^{S}(x, A)=\inf \left\{p^{S}(x, a): a \in A\right\}$.

Lemma B [35] Let $(X, p)$ be a partial metric space and $A$ be a non-empty subset of $X$, then $a \in \bar{A}$ if and only if $p(a, A)=p(a, a)$.

Proposition 1.4 [29] Let $(X, p)$ be a partial metric space. For any $A, B, C \in C B^{p}(X)$, we have the following:

(i) $\delta_{p}(A, A)=\sup \{p(a, a): a \in A\}$;

(ii) $\delta_{p}(A, A) \leq \delta_{p}(A, B)$;

(iii) $\delta_{p}(A, B)=0$ implies $A \subseteq B$;

(iv) $\delta_{p}(A, B) \leq \delta_{p}(A, C)+\delta_{p}(C, B)-\inf _{c \in C} p(c, c)$.

Proposition 1.5 [29] Let $(X, p)$ be a partial metric space. For any $A, B, C \in C B^{p}(X)$, we have the following:

(h1) $H_{p}(A, A) \leq H_{p}(A, B)$;

(h2) $H_{p}(A, B)=H_{p}(B, A)$;

(h3) $H_{p}(A, B) \leq H_{p}(A, C)+H_{p}(C, B)-\inf _{c \in C} p(c, c)$;

(h4) $H_{p}(A, B)=0$ implies that $A=B$.

The mapping $H_{p}: C B^{p}(X) \times C B^{p}(X) \rightarrow[0, \infty)$ is called a partial Hausdorff metric induced by a partial metric $p$. Every Hausdorff metric is a partial Hausdorff metric, but the converse is not true (see Example 2.6 in [29]).

Lemma C [29] Let $(X, p)$ be a partial metric space and $A, B \in C B^{p}(X)$ and $h>1$, then for any $a \in A$, there exists $a b \in$ such that $p(a, b) \leq h H_{p}(A, B)$.

Theorem 1.6 [29] Let $(X, p)$ be a partial metric space. If $T: X \rightarrow C B^{p}(X)$ is a multi-valued mapping such that for all $x, y \in X$, we have $H_{p}(T x, T y) \leq k p(x, y)$, where $k \in(0,1)$. Then $T$ has a fixed point. 
Definition 1.7 Let $(X, p)$ be a partial metric space and $f: X \rightarrow X$ and $T: X \rightarrow C B^{p}(X)$. A point $x \in X$ is said to be (i) a fixed point of $f$ if $x=f(x)$, (ii) a fixed point of $T$ if $x \in T(x)$, (iii) a coincidence point of a pair $(f, T)$ if $f x \in T x$, (iv) a common fixed point of the pair $(f, T)$ if $x=f x \in T x$.

We denote the set of all fixed points of $f$, the set of all coincidence points of the pair $(f, T)$ and the set of all common fixed points of the pair $(f, T)$ by $F(f), C(f, T)$ and $F(f, T)$, respectively. Motivated by the work of $[4,13]$, we give the following definitions in partial metric spaces.

Definition 1.8 Let $(X, p)$ be a partial metric space and $f: X \rightarrow X$ and $T: X \rightarrow C B^{p}(X)$. The pair $(f, T)$ is called (i) commuting if $T f x=f T x$ for all $x \in X$, (ii) weakly compatible if the pair $(f, T)$ commutes at their coincidence points, that is, $f T x=T f x$ whenever $x \in C(f, T)$, (iii) $I T$-commuting [11] at $x \in X$ if $f T x \subseteq T f x$.

Definition 1.9 Let $(X, p)$ be a partial metric space and $Y$ be any non-empty set. Let $f$ : $Y \rightarrow X$ and $T: Y \longrightarrow C B^{p}(X)$ be single-valued and multi-valued mappings, respectively. Suppose that $x_{0} \in Y$, then the set

$$
O\left(f, T ; x_{0}\right)=\left\{y_{n}: y_{n+1}=f x_{n+1} \in T x_{n} \text { for } n=0,1,2, \ldots\right\}
$$

is called an orbit for the pair $(f, T)$ at $x_{0}$. A partial metric space $X$ is called $(f, T)$-orbitally complete if and only if every Cauchy sequence in the orbit for $(f, T)$ at $x_{0}$ converges with respect to $\tau_{p}$ to a point $x \in X$ such that $p(x, x)=\lim _{n, m \rightarrow \infty} p\left(x_{m}, x_{n}\right)$.

Singh and Mishra [13] introduced Suzuki-Zamfirescu type hybrid contractive condition in complete metric spaces. In the context of partial metric spaces, the condition is given as follows.

Definition 1.10 Let $(X, p)$ be a partial metric space, $f: Y \longrightarrow X$ and $T: Y \longrightarrow C B^{p}(X)$ be single-valued and multi-valued mappings, respectively. The hybrid pair $(f, T)$ is said to satisfy Suzuki-Zamfirescu hybrid contraction condition if there exists $r \in[0,1)$ such that $\omega(r) p(f x, T x) \leq p(f x, f y)$ implies that

$$
H_{p}(T x, T y) \leq r M_{p, f}(x, y)
$$

for all $x, y \in Y$ and

$$
M_{p, f}(x, y)=\max \left\{p(f x, f y), \frac{p(f x, T x)+p(f y, T y)}{2}, \frac{p(f x, T y)+p(f y, T x)}{2}\right\} .
$$

Lemma $\mathbf{D}$ Let $(X, p)$ be a partial metric space, $f: Y \rightarrow X$ and $T: Y \rightarrow C B^{p}(X)$ be singlevalued and multi-valued mappings, respectively. Then the partial metric space $(X, p)$ is $(f, T)$-orbitally complete if and only if $\left(X, p^{S}\right)$ is $(f, T)$-orbitally complete.

Proof Suppose that $\left(X, p^{S}\right)$ is $(f, T)$-orbitally complete and $x_{0}$ is an arbitrary element of $X$. If $\left\{y_{n}\right\}$ is a Cauchy sequence in $O\left(f, T ; x_{0}\right)$ in $(X, p)$, then it is also Cauchy in $\left(X, p^{S}\right)$. There- 
fore, by (1.2) we deduce that there exists $y$ in $X$ such that

$$
p(y, y)=\lim _{n \rightarrow \infty} p\left(y, y_{n}\right)=\lim _{n, m \rightarrow \infty} p\left(y_{n}, y_{m}\right),
$$

and $\left\{y_{n}\right\}$ converges to $y$ in $(X, p)$. Conversely, let $(X, p)$ be $(f, T)$-orbitally complete. If $\left\{y_{n}\right\}$ is a Cauchy sequence in $O\left(f, T ; x_{0}\right)$ in $\left(X, p^{S}\right)$, then it is also a Cauchy sequence in $(X, p)$. Therefore,

$$
p(y, y)=\lim _{n \rightarrow \infty} p\left(y, y_{n}\right)=\lim _{n, m \rightarrow \infty} p\left(y_{n}, y_{m}\right) .
$$

For given $\varepsilon>0$, there exists $n_{\varepsilon} \in \mathbb{N}$ such that

$$
\left|p\left(y, y_{n}\right)-p(y, y)\right|<\frac{\varepsilon}{2} \quad \text { and } \quad\left|p\left(y, y_{n}\right)-p\left(y_{n}, y_{m}\right)\right|<\frac{\varepsilon}{2}
$$

for all $m, n>n_{\varepsilon}$. Consequently, we have

$$
\begin{aligned}
p^{S}\left(y, y_{n}\right) & =2 p\left(y, y_{n}\right)-p(y, y)-p\left(y_{n}, y_{m}\right) \\
& \leq\left|p\left(y, y_{n}\right)-p(y, y)+p\left(y, y_{n}\right)-p\left(y_{n}, y_{m}\right)\right| \\
& \leq\left|p\left(y, y_{n}\right)-p(y, y)\right|+\left|p\left(y, y_{n}\right)-p\left(y_{n}, y_{m}\right)\right| \\
& <\frac{\varepsilon}{2}+\frac{\varepsilon}{2}=\varepsilon,
\end{aligned}
$$

whenever $m, n>n_{\varepsilon}$. The result follows.

\section{Coincidence points of a hybrid pair of mappings}

In the following theorem, the existence of coincidence points of a hybrid pair of singlevalued and multi-valued mappings that satisfy Suzuki-Zamfirescu hybrid contraction condition in partial metric spaces is established.

Theorem 2.1 Let $(X, p)$ be a partial metric space and $Y$ be any non-empty set. Assume that a pair of mappings $f: Y \rightarrow X$ and $T: Y \longrightarrow C B^{p}(X)$ satisfies Suzuki-Zamfirescu hybrid contraction condition with $T(Y) \subset f(Y)$. If there exists $u_{0} \in Y$ such that $f(Y)$ is $(f, T)$-orbitally complete at $u_{0}$, then $C(f, T) \neq \phi$. If $Y=X$ and $(f, T)$ is IT-commuting at coincidence points of $(f, T)$, then $F(f, T) \neq \phi$ provided that $f z$ is a fixed point of for some $z \in C(f, T)$.

Proof Let $h=1 / \sqrt{r}$ and $u_{0} \in Y$ be such that $y_{0}=f u_{0}$. By the given assumption, we have $T u_{0} \subseteq f(Y)$. So, there exists a point $u_{1} \in Y$ such that $y_{1}=f u_{1} \in T u_{0}$. As $h>1$, so by Lemma $C$, there exists a point $y_{2} \in T u_{1}$ such that

$$
p\left(f u_{1}, y_{2}\right) \leq h H_{p}\left(T u_{0}, T u_{1}\right) .
$$

Using the fact that $T u_{1} \subseteq f(Y)$, we obtain a point $u_{2} \in Y$ such that $y_{2}=f u_{2} \in T u_{1}$. Therefore,

$$
p\left(f u_{1}, f u_{2}\right) \leq h H_{p}\left(T u_{0}, T u_{1}\right) .
$$


Since

$$
\omega(r) p\left(f u_{0}, T u_{0}\right) \leq \omega(r) p\left(f u_{0}, f u_{1}\right) \leq p\left(f u_{0}, f u_{1}\right)
$$

we have

$$
\begin{aligned}
p\left(f u_{1}, f u_{2}\right) & \leq h H_{p}\left(T u_{0}, T u_{1}\right) \\
& \leq h r \max \left\{p\left(f u_{0}, f u_{1}\right), \frac{p\left(f u_{0}, T u_{0}\right)+p\left(f u_{1}, T u_{1}\right)}{2}, \frac{p\left(f u_{0}, T u_{1}\right)+p\left(f u_{1}, T u_{0}\right)}{2}\right\} \\
& \leq \frac{1}{\sqrt{r}} r \max \left\{p\left(y_{0}, y_{1}\right), \frac{p\left(y_{0}, y_{1}\right)+p\left(y_{1}, y_{2}\right)}{2}, \frac{p\left(y_{0}, y_{2}\right)+p\left(y_{1}, y_{1}\right)}{2}\right\} \\
& \leq \sqrt{r} \max \left\{p\left(y_{0}, y_{1}\right), \frac{p\left(y_{0}, y_{1}\right)+p\left(y_{1}, y_{2}\right)}{2}\right\} .
\end{aligned}
$$

If

$$
\max \left\{p\left(y_{0}, y_{1}\right), \frac{p\left(y_{0}, y_{1}\right)+p\left(y_{1}, y_{2}\right)}{2}\right\}=p\left(y_{0}, y_{1}\right)
$$

then

$$
p\left(y_{1}, y_{2}\right) \leq h H_{p}\left(T u_{0}, T u_{1}\right) \leq \sqrt{r} p\left(y_{0}, y_{1}\right)
$$

If

$$
\max \left\{p\left(y_{0}, y_{1}\right), \frac{p\left(y_{0}, y_{1}\right)+p\left(y_{1}, y_{2}\right)}{2}\right\}=\frac{p\left(y_{0}, y_{1}\right)+p\left(y_{1}, y_{2}\right)}{2}
$$

then we obtain

$$
p\left(y_{1}, y_{2}\right) \leq \frac{\sqrt{r}}{2-\sqrt{r}} p\left(y_{0}, y_{1}\right) \leq \sqrt{r} p\left(y_{0}, y_{1}\right) .
$$

As $f u_{2} \in T u_{1}$, we choose $y_{3} \in T u_{2}$ such that $p\left(f u_{2}, y_{3}\right) \leq h H\left(T u_{1}, T u_{2}\right)$. Using the fact that $T u_{2} \subseteq f(Y)$, we obtain a point $u_{3} \in Y$ such that $y_{3}=f u_{3} \in T u_{2}$ and

$$
p\left(f u_{2}, f u_{3}\right) \leq h H_{p}\left(T u_{1}, T u_{2}\right)
$$

Since

$$
\omega(r) p\left(f u_{1}, T u_{1}\right) \leq \omega(r) p\left(f u_{1}, f u_{2}\right) \leq p\left(f u_{1}, f u_{2}\right)
$$

so we have

$$
\begin{aligned}
p\left(f u_{2}, f u_{3}\right) & \leq h H_{p}\left(T u_{1}, T u_{2}\right) \\
& \leq h r \max \left\{p\left(f u_{1}, f u_{2}\right), \frac{p\left(f u_{1}, T u_{1}\right)+p\left(f u_{2}, T u_{2}\right)}{2}, \frac{p\left(f u_{1}, T u_{2}\right)+p\left(f u_{2}, T u_{1}\right)}{2}\right\}
\end{aligned}
$$




$$
\begin{aligned}
& \leq \frac{1}{\sqrt{r}} r \max \left\{p\left(y_{1}, y_{2}\right), \frac{p\left(y_{1}, y_{2}\right)+p\left(y_{2}, y_{3}\right)}{2}, \frac{p\left(y_{1}, y_{3}\right)+p\left(y_{2}, y_{2}\right)}{2}\right\} \\
& \leq \sqrt{r} \max \left\{p\left(y_{1}, y_{2}\right), \frac{p\left(y_{1}, y_{2}\right)+p\left(y_{2}, y_{3}\right)}{2}\right\} .
\end{aligned}
$$

Following the arguments similar to those given above, we obtain

$$
p\left(y_{2}, y_{3}\right) \leq \sqrt{r} p\left(y_{1}, y_{2}\right)
$$

which further implies that

$$
p\left(y_{2}, y_{3}\right) \leq(\sqrt{r})^{2} p\left(y_{0}, y_{1}\right) .
$$

Continuing this process, we obtain a sequence $\left\{y_{n}\right\} \subset Y$ such that for any integer $n \geq 0$, $y_{n+1}=f u_{n+1} \in T u_{n}$ and

$$
p\left(y_{n}, y_{n+1}\right) \leq(\sqrt{r})^{n} p\left(y_{0}, y_{1}\right)
$$

for every $n \in \mathbb{N}$. This shows that $\lim _{n \rightarrow \infty} p\left(y_{n}, y_{n+1}\right)=0$. Since

$$
p\left(y_{n}, y_{n}\right)+p\left(y_{n+1}, y_{n+1}\right) \leq 2 p\left(y_{n}, y_{n+1}\right)
$$

so we obtain

$$
\lim _{n \rightarrow \infty} p\left(y_{n}, y_{n}\right)=0 \quad \text { and } \quad \lim _{n \rightarrow \infty} p\left(y_{n+1}, y_{n+1}\right)=0 .
$$

Now, for $m>n \geq 1$, we have

$$
\begin{aligned}
p^{S}\left(y_{n}, y_{n+m}\right) & =2 p\left(y_{n}, y_{n+m}\right)-p\left(y_{n}, y_{n}\right)-p\left(y_{n+m}, y_{n+m}\right) \\
& \leq 2 p\left(y_{n}, y_{n+1}\right)+2 p\left(y_{n+1}, y_{n+2}\right)+\cdots+2 p\left(y_{n+m-1}, y_{n+m}\right) \\
& \leq 2\left((\sqrt{r})^{n}+(\sqrt{r})^{n+1}+\cdots+(\sqrt{r})^{n+m-1}\right) p\left(y_{0}, y_{1}\right) .
\end{aligned}
$$

It follows that $\left\{y_{n}\right\}$ is a Cauchy sequence in $\left(f(Y), p^{S}\right)$. By Lemma $\mathrm{A}$, we have $\left\{y_{n}\right\}$ is a Cauchy sequence in $(f(Y), p)$. Since $(f(Y), p)$ is $(f, T)$-orbitally complete at $u_{0}$, so again by Lemma $\mathrm{D},\left(f(Y), p^{S}\right)$ is $(f, T)$-orbitally complete at $u_{0}$. Hence, there exists an element $u \in f(Y)$ such that $\lim _{n \rightarrow \infty} p^{S}\left(y_{n}, y\right)=0$. This implies that

$$
\lim _{n \rightarrow \infty} p\left(y_{n}, u\right)=\lim _{n \rightarrow \infty} p\left(y_{n}, y_{n}\right)=p(u, u)=0 .
$$

Let $z \in f^{-1} u$, then $z \in Y$ and $u=f z$. Now,

$$
\begin{aligned}
& p(f z, T x) \leq p\left(f z, f u_{n+1}\right)+p\left(f u_{n+1}, T x\right)-p\left(f u_{n+1}, f u_{n+1}\right) \quad \text { and } \\
& p\left(f u_{n+1}, T x\right) \leq p\left(f u_{n+1}, f u_{n}\right)+p\left(f u_{n}, f z\right)+p(f z, T x)-p\left(f u_{n}, f u_{n}\right)-p(f z, f z)
\end{aligned}
$$

give

$$
\lim _{n \rightarrow \infty} p\left(f u_{n+1}, T x\right)=p(f z, T x) .
$$


Similarly, we can show that

$$
\lim _{n \rightarrow \infty} p\left(f u_{n}, T x\right)=p(f z, T x)
$$

Now, we will claim that

$$
p(f z, T x) \leq r p(f z, f x) \quad \text { for any } f x \in f(Y)-\{f z\} .
$$

If $x=z$ or $f x=f z$, then $p(f x, T x)=0$. This gives $p^{S}(f x, T x)=0$, which implies that $f x \in T x$ and we are done. Now from (2.1), there exists a positive integer $n_{0}$ such that for all $n \geq n_{0}$,

$$
p\left(f z, f u_{n+1}\right) \leq \frac{1}{3} p(f z, f x) \quad \text { and } \quad p\left(f z, f u_{n}\right) \leq \frac{1}{3} p(f z, f x)
$$

So, for any $n \geq n_{0}$, we have

$$
\begin{aligned}
\omega(r) p\left(f u_{n}, T u_{n}\right) & \leq p\left(f u_{n}, T u_{n}\right) \leq p\left(f u_{n}, f u_{n+1}\right) \\
& \leq p\left(f u_{n}, f z\right)+p\left(f z, f u_{n+1}\right)-p(f z, f z) \leq \frac{2}{3} p(f z, f x) \\
& \leq p(f z, f x)-\frac{1}{3} p(f z, f x) \leq p(f z, f x)-p\left(f z, f u_{n}\right) \\
& \leq p\left(f u_{n}, f x\right)-p\left(f u_{n}, f u_{n}\right) \leq p\left(f u_{n}, f x\right) .
\end{aligned}
$$

Hence, for any $n \geq n_{0}$, we obtain

$$
\omega(r) p\left(f u_{n}, T u_{n}\right) \leq p\left(f u_{n}, f x\right)
$$

This implies

$$
\begin{aligned}
p\left(f u_{n+1}, T x\right) \leq & H_{p}\left(T u_{n}, T x\right) \\
\leq & r \max \left\{p\left(f u_{n}, f x\right), \frac{p\left(f u_{n}, T u_{n}\right)+p(f x, T x)}{2}, \frac{p\left(f u_{n}, T x\right)+p\left(f x, T u_{n}\right)}{2}\right\} \\
\leq & r \max \left\{p\left(y_{n}, f x\right), \frac{p\left(y_{n}, y_{n+1}\right)+p(f x, T x)}{2}, \frac{p\left(y_{n}, T x\right)+p\left(f x, y_{n+1}\right)}{2}\right\} \\
\leq & r \max \left\{p\left(y_{n}, u\right)+p(u, f x)-p(u, u), \frac{p\left(y_{n}, y_{n+1}\right)+p(f x, T x)}{2},\right. \\
& \left.\frac{p\left(y_{n}, u\right)+p(u, T x)-p(u, u)+p(f x, u)+p\left(u, y_{n+1}\right)-p(u, u)}{2}\right\} .
\end{aligned}
$$

On taking limit as $n$ tends to $\infty$, we obtain

$$
\begin{aligned}
p(f z, T x) & \leq r \max \left\{p(u, f x), \frac{p(f x, T x)}{2}, \frac{p(u, T x)+p(f x, u)}{2}\right\} \\
& =r \max \left\{p(f z, f x), \frac{p(f x, T x)}{2}, \frac{p(f z, T x)+p(f x, f z)}{2}\right\} \\
& \leq r \max \left\{p(f z, f x), \frac{p(f z, T x)+p(f x, f z)}{2}\right\} .
\end{aligned}
$$


If

$$
\max \left\{p(f z, f x), \frac{p(f z, T x)+p(f x, f z)}{2}\right\}=p(f z, f x),
$$

then we are done. If

$$
\max \left\{p(f z, f x), \frac{p(f z, T x)+p(f x, f z)}{2}\right\}=\frac{p(f z, T x)+p(f x, f z)}{2},
$$

then we obtain

$$
p(f z, T x) \leq \frac{r}{2-r} p(f x, f z) \leq r p(f x, f z)
$$

and hence (2.2) holds. Next, we show that

$$
H_{p}(T z, T x) \leq r \max \left\{p(f z, f x), \frac{p(f x, T x)+p(f z, T z)}{2}, \frac{p(f x, T z)+p(f z, T x)}{2}\right\}
$$

for any $x \in Y$. If $x=z$, then $f x=f z$, and the claim follows from (2.2). Suppose that $x \neq z$, then $f x \neq f z$. As $f$ is a non-constant single-valued mapping, we have

$$
\begin{aligned}
p(f x, T x) & \leq p(f x, f z)+p(f z, T x)-p(f z, f z) \\
& \leq p(f x, f z)+r p(f x, f z) \leq(1+r) p(f x, f z) .
\end{aligned}
$$

This implies

$$
\omega(r) p(f x, T x) \leq p(f x, f z)
$$

Therefore,

$$
H_{p}(T z, T x) \leq r \max \left\{p(f z, f x), \frac{p(f x, T x)+p(f z, T z)}{2}, \frac{p(f x, T z)+p(f z, T x)}{2}\right\} .
$$

Hence, (2.3) holds for any $x \in Y$. Note that

$$
\begin{aligned}
p\left(T z, f u_{n+2}\right) \leq & H_{p}\left(T z, T u_{n+1}\right) \\
\leq & r \max \left\{p\left(f z, f u_{n+1}\right), \frac{p\left(f u_{n+1}, T u_{n+1}\right)+p(f z, T z)}{2},\right. \\
& \left.\frac{p\left(f u_{n+1}, T z\right)+p\left(f z, T u_{n+1}\right)}{2}\right\} \\
\leq & r \max \left\{p\left(f z, y_{n+2}\right), \frac{p\left(y_{n+2}, y_{n+2}\right)+p(f z, T z)}{2},\right. \\
& \left.\frac{p\left(y_{n+2}, f z\right)+p(f z, T z)-p(f z, f z)+p\left(f z, y_{n+2}\right)}{2}\right\} .
\end{aligned}
$$

On taking limit as $n \rightarrow \infty$, we obtain

$$
p(f z, T z) \leq \frac{r}{2} p(f z, T z)
$$


We obtain $p(f z, T z)=0$, which further implies that $p^{S}(f z, T z) \leq 2 p(f z, T z)=0$. Hence, $f z \in$ $T z$. Further if $Y=X$ and $f f z=f z$, then due to $I T$-commutativity of the pair $(f, T)$, we have $f z=f f z \in f T z \subseteq T f z$. This shows that $f z$ is a common fixed point of the pair $(f, T)$.

Corollary A Let $(X, p)$ be a partial metric space and $Y$ be any non-empty set. Assume that here exists $r \in[0,1)$ such that the mappings $f: Y \rightarrow X$ and $T: Y \rightarrow C B^{p}(X)$ satisfy

$$
\omega(r) p(f x, T x) \leq p(f x, f y) \quad \Rightarrow \quad H_{p}(T x, T y) \leq r p(f x, f y)
$$

for all $x, y \in Y$, with $T(Y) \subset f(Y)$. If there exists $u_{0} \in Y$ such that $f(Y)$ is $(f, T)$-orbitally complete at $u_{0}$, then $C(f, T) \neq \phi$. If $Y=X$ and $(f, T)$ is IT-commuting at coincidence points of the pair $(f, T)$, then $F(f, T) \neq \phi$ provided that $f z$ is a fixed point off for some $z \in C(f, T)$.

Example 2.2 Let $X=\{0,1,2\}$ and $Y=\{0,1,2,3\}$. Define a mapping $p: X \times X \rightarrow \mathbb{R}^{+}$as follows:

$$
\begin{aligned}
& p(0,0)=p(1,1)=0, \quad p(0,1)=p(1,0)=\frac{1}{4}, \quad p(2,2)=\frac{1}{3}, \\
& p(0,2)=p(2,0)=\frac{2}{5}, \quad p(1,2)=p(2,1)=\frac{13}{20} .
\end{aligned}
$$

Then $p$ is a partial metric on $X$. Let $\omega(r)$ be as given in Theorem 2.1 and the mappings $T: Y \rightarrow C B^{p}(X)$ and $f: Y \rightarrow X$ be given as

$$
T x=\left\{\begin{array}{ll}
\{0\} & \text { when } x \neq 2, \\
\{0,1\} & \text { when } x=2,
\end{array} \text { and } f x= \begin{cases}0, & \text { if } x \in\{0,1\} \\
2, & \text { if } x=2, \\
1, & \text { if } x=3 .\end{cases}\right.
$$

Note that

$$
\begin{aligned}
& p(f 0, T 0)=p(f 1, T 1)=p(f 0, f 1)=p(f 1, f 1)=0, \\
& p(f 3, f 2)=\frac{13}{20}, \quad p(f 2, f 2)=\frac{1}{3}, \\
& p(f 1, f 2)=p(f 0, f 2)=p(f 2, T 2)=\frac{2}{5}, \\
& p(f 3, T 3)=p(f 3, f 0)=p(f 3, f 1)=\frac{1}{4} .
\end{aligned}
$$

If we take $r \geq \frac{3}{5}$ and $\omega(r) \leq \frac{5}{8}$, then for all $x, y \in Y$,

$$
\omega(r) p(f x, T x) \leq p(f x, f y)
$$

holds. If we consider $r=\frac{5}{6}$, then $\omega(r)=\frac{1}{6}$. Then, for $x, y \in\{0,1,3\}$, we have $H_{p}(T x, T y)=0$, hence $H_{p}(T x, T y) \leq r p(f x, f y)$ is satisfied trivially. Now consider

$$
\begin{aligned}
& H_{p}(T 0, T 2)=p(0,1)=\frac{1}{4} \leq \frac{1}{3}=r p(f 0, f 2), \\
& H_{p}(T 1, T 2)=p(0,1)=\frac{1}{4} \leq \frac{1}{3}=r p(f 1, f 2),
\end{aligned}
$$




$$
\begin{aligned}
& H_{p}(T 2, T 3)=p(0,1)=\frac{1}{4}<\frac{13}{24}=r p(f 2, f 3), \\
& H_{p}(T 2, T 2)=p(0,1)=\frac{1}{4}<\frac{5}{18}=r p(f 2, f 2) .
\end{aligned}
$$

Hence, for all $x, y \in Y$,

$$
\omega(r) p(f x, T x) \leq p(f x, f y)
$$

implies

$$
H_{p}(T x, T y) \leq r p(f x, f y)
$$

Let $u_{0}=1, y_{0}=f(1)=0$. As $T(0) \subseteq f(Y)$, there exists a point $u_{1}=1$ in $Y$ such that $y_{1}=f(1)=$ $0 \in T(1)$ and $T(0)=\{0\} \subseteq f(Y)$, we obtain a point $u_{2}=1$ in $Y$ such that $y_{2}=0=f(1) \in T(1)$. Continuing this way, we construct an orbit $\left\{y_{0}=y_{1}=y_{2}=\cdots=0\right\}$ for $(f, T)$ at $u_{0}=1$. Also, $f(Y)$ is $(f, T)$-orbitally complete at $u_{0}=0$. So, all the conditions of Corollary A are satisfied. Moreover, $C(f, T)=\{0,1\}$.

On the other hand, the metric $p^{S}$ induced by the partial metric $p$ is given by

$$
\begin{aligned}
& p^{S}(0,0)=p^{S}(1,1)=p^{S}(2,2)=0, \\
& p^{S}(0,1)=p^{S}(1,0)=\frac{1}{2}, \quad p^{S}(1,2)=p^{S}(2,1)=\frac{29}{30}, \\
& p^{S}(0,2)=p^{S}(2,0)=\frac{7}{15} .
\end{aligned}
$$

Now, we show that Corollary A is not applicable (in the case of a metric induced by a partial metric $p$ ) in this case. Since

$$
\omega(r) p^{S}(f 1, T 1)=\omega(r) p^{S}(0,0)=0 \leq p^{S}(f x, f y)
$$

is satisfied for any $r \in[0,1), x$ and $y$ in $X$, so it must imply $H_{p^{S}}(T 1, T 2) \leq r p(f 1, f 2)$. But

$$
H_{p^{S}}(T 1, T 2)=H_{p} s(\{0\},\{0,1\})=\frac{1}{2}
$$

and

$$
p^{S}(f 1, f 2)=p^{S}(0,2)=\frac{7}{15}<\frac{1}{2}
$$

Hence, for any $r \in[0,1)$,

$$
H_{p^{s}}(T 1, T 2) \nless r p(f 1, f 2) .
$$

Corollary B Let $(X, p)$ be a partial metric space, $Y$ be any non-empty set and $f, T: Y \longrightarrow X$ be such that $T(Y) \subset f(Y)$. Suppose that there exists $u_{0} \in Y$ such that $f(Y)$ is $(f, T)$-orbitally complete at $u_{0}$. Assume further that there exists an $r \in[0,1)$ such that

$\omega(r) p(f x, T x) \leq p(f x, f y)$ 
implies that

$$
p(T x, T y) \leq r \max \left\{p(f x, f y), \frac{p(f x, T x)+p(f y, T y)}{2}, \frac{p(f x, T y)+p(f y, T x)}{2}\right\}
$$

for all $x, y \in Y$. Then $C(f, T) \neq \phi$. Further, if $Y=X$ and the pair $(f, T)$ is commuting at $x$ where $x \in C(f, T)$, then $F(f, T)$ is a singleton.

Proof It follows from Theorem 2.1, that $C(f, T) \neq \phi$. If $u \in C(f, T)$, then $f u=T u$. Further, if $Y=X$ and $(f, T)$ is commuting at $u$, then $f f u=f T u=T f u$. Now,

$$
\omega(r) p(f u, T f u) \leq p(f u, T f u)=p(f u, f f u)
$$

implies that

$$
\begin{aligned}
p(f u, f f u) & =p(T u, T f u) \\
& \leq r M_{p, f}(u, f u) \\
& \leq r \max \left\{p(f u, f f u), \frac{p(f f u, T f u)+p(f u, T u)}{2}, \frac{p(f f u, T u)+p(f u, T f u)}{2}\right\} \\
& \leq r \max \left\{p(f u, f f u), \frac{p(f f u, f f u)+p(f u, T u)}{2}, \frac{p(f f u, f u)+p(f u, f f u)}{2}\right\} \\
& \leq r \max \left\{p(f u, f f u), \frac{p(f f u, f u)+p(f u, f f u)}{2}, \frac{p(f f u, f u)+p(f u, f f u)}{2}\right\} \\
& \leq r p(f u, f f u) .
\end{aligned}
$$

As $r<1$, we obtain $p(f u, f f u)=0$, which further implies that $p^{S}(f u, f f u) \leq 2 p(f u, f f u)=0$. Hence, $f u$ is a common fixed point of $f$ and $T$.

For uniqueness, assume there exist $z_{1} \neq z_{2}$, such that $z_{1}=f z_{1}=T z_{1}$ and $z_{2}=f z_{2}=T z_{2}$. Then

$$
\psi(r) p\left(f z_{1}, T z_{1}\right) \leq p\left(f z_{1}, T z_{1}\right)=p\left(f z_{1}, f z_{1}\right) \leq p\left(f z_{1}, f z_{2}\right),
$$

which implies

$$
\begin{aligned}
p\left(z_{1}, z_{2}\right) & =p\left(T z_{1}, T z_{2}\right) \\
& \leq r \max \left\{p\left(f z_{1}, f z_{2}\right), \frac{p\left(f z_{1}, T z_{1}\right)+p\left(f z_{2}, T z_{2}\right)}{2}, \frac{p\left(f z_{2}, T z_{1}\right)+p\left(f z_{1}, T z_{2}\right)}{2}\right\} \\
& \leq r \max \left\{p\left(z_{1}, z_{2}\right), p\left(z_{1}, z_{2}\right), p\left(z_{1}, z_{2}\right)\right\} \\
& \leq r p\left(z_{1}, z_{2}\right) .
\end{aligned}
$$

We obtain $p\left(z_{1}, z_{2}\right)=0$, which further implies that $p^{S}\left(z_{1}, z_{2}\right) \leq 2 p\left(z_{1}, z_{2}\right)=0$. Hence, $z_{1}=z_{2}$. 


\section{An application}

In this section, we assume that $U$ and $V$ are Banach spaces, $W \subseteq U$ and $D \subseteq V$. Suppose that

$$
\begin{aligned}
& \tau: W \times D \longrightarrow W, \\
& g, g^{\prime}, h, h^{\prime}: W \times D \longrightarrow \mathbb{R}, \\
& G, F: W \times D \times \mathbb{R} \longrightarrow \mathbb{R} .
\end{aligned}
$$

Considering $W$ and $D$ as the state and decision spaces respectively, the problem of dynamic programming reduces to the problem of solving the functional equations:

$$
\begin{array}{ll}
p(x)=\sup _{y \in D}\{h(x, y)+G(x, y, p(\tau(x, y)))\}, & \text { for } x \in W, \\
q(x)=\sup _{y \in D}\left\{h^{\prime}(x, y)+F(x, y, q(\tau(x, y)))\right\}, & \text { for } x \in W .
\end{array}
$$

Then equations (3.1) and (3.2) can be reformulated as

$$
\begin{aligned}
& p(x)=\sup _{y \in D}\{g(x, y)+G(x, y, p(\tau(x, y)))\}-b, \quad \text { for } x \in W, \\
& q(x)=\sup _{y \in D}\left\{g^{\prime}(x, y)+F(x, y, q(\tau(x, y)))\right\}-b, \quad \text { for } x \in W .
\end{aligned}
$$

For more on the multistage process involving such functional equations, we refer to [23, 31-34]. Now, we study the existence and uniqueness of a common and bounded solution of the functional equations (3.3)-(3.4) arising in dynamic programming in the setup of partial metric spaces.

Let $B(W)$ denote the set of all bounded real-valued functions on $W$. For an arbitrary $h \in B(W)$, define $\|h\|=\sup _{x \in W}|h(x)|$. Then $(B(W),\|\cdot\|)$ is a Banach space endowed with the metric $d$ defined as $d(h, k)=\sup _{x \in W}|h x-k x|$. Now, consider

$$
p_{B}(h, k)=d(h, k)+b=\sup _{x \in W}|h x-k x|+b
$$

where $h, k \in B(W), b>0$ and $p_{B}$ is a partial metric on $B(W)$. Let $\omega(r)$ be defined as in Section 1. Suppose that the following conditions hold:

(C1): $G, F, g$, and $g^{\prime}$ are bounded.

(C2): For $x \in W, h \in B(W)$ and $b>0$, define

$$
\begin{aligned}
& K h(x)=\sup _{y \in D}\{g(x, y)+G(x, y, h(\tau(x, y)))\}-b, \\
& J h(x)=\sup _{y \in D}\left\{g^{\prime}(x, y)+F(x, y, h(\tau(x, y)))\right\}-b .
\end{aligned}
$$

Moreover, assume that there exists $r \in[0,1)$ such that for every $(x, y) \in W \times D, h, k \in B(W)$ and $t \in W$,

$$
\omega(r) p_{B}(K h(t), J h(t)) \leq p_{B}(J h(t), J k(t))
$$


implies

$$
|G(x, y, h(t))-G(x, y, k(t))| \leq r M_{p_{B},}(h(t), k(t)),
$$

where

$$
\begin{aligned}
M_{p_{B}, J}(h(t), k(t))= & \max \left\{p_{B}(J h(t), J k(t)), \frac{p_{B}(J k(t), K k(t))+p_{B}(J h(t), K h(t))}{2},\right. \\
& \left.\frac{p_{B}(J h(t), K k(t))+p_{B}(J k(t), K h(t))}{2}\right\} .
\end{aligned}
$$

(C3): For any $h \in B(W)$, there exists $k \in B(W)$ such that for $x \in W$,

$$
K h(x)=J k(x) .
$$

(C4): There exists $h \in B(W)$ such that

$$
K h(x)=J h(x) \quad \text { implies that } J K h(x)=K J h(x) .
$$

Theorem 3.1 Assume that the conditions (C1)-(C4) are satisfied. IfJ $(B(W))$ is a closed convex subspace of $B(W)$, then the functional equations (3.3) and (3.4) have a unique, common and bounded solution.

Proof Note that $\left(B(W), p_{B}\right)$ is a complete partial metric space. By $(\mathrm{C} 1), J, K$ are self-maps of $B(W)$. The condition (C3) implies that $K(B(W)) \subseteq J(B(W))$. It follows from (C4) that $J$ and $K$ commute at their coincidence points. Let $\lambda$ be an arbitrary positive number and $h_{1}, h_{2} \in B(W)$. Choose $x \in W$ and $y_{1}, y_{2} \in D$ such that

$$
K h_{j}<g\left(x, y_{j}\right)+G\left(x, y_{j}, h_{j}\left(x_{j}\right)-b+\lambda,\right.
$$

where $x_{j}=\tau\left(x, y_{j}\right), j=1,2$. Further, from (3.5) and (3.6), we have

$$
\begin{aligned}
& K h_{1} \geq g\left(x, y_{2}\right)+G\left(x, y_{2}, h_{1}\left(x_{2}\right)\right), \\
& K h_{2} \geq g\left(x, y_{1}\right)+G\left(x, y_{1}, h_{2}\left(x_{1}\right)\right) .
\end{aligned}
$$

Therefore, (3.8) in (C2) becomes

$$
\omega(r) p_{B}\left(K h_{1}(x), J h_{1}(x)\right) \leq p_{B}\left(J h_{1}(x)-J h_{2}(x)\right) .
$$

Then (3.13) together with (3.10) and (3.12) implies

$$
\begin{aligned}
K h_{1}(x)-K h_{2}(x) & <G\left(x, y_{1}, h_{1}\left(x_{1}\right)\right)-G\left(x, y_{1}, h_{2}\left(x_{2}\right)\right)-b+\lambda \\
& \leq\left|G\left(x, y_{1}, h_{1}\left(x_{1}\right)\right)-G\left(x, y_{1}, h_{2}\left(x_{2}\right)\right)\right|-b+\lambda \\
& \leq r M_{p_{B},}(h(t), k(t))-b+\lambda .
\end{aligned}
$$


Now, (3.10), (3.11) and (3.13) imply

$$
\begin{aligned}
K h_{2}(x)-K h_{1}(x) & \leq G\left(x, y_{1}, h_{2}\left(x_{2}\right)\right)-G\left(x, y_{1}, h_{1}\left(x_{1}\right)\right)-b \\
& \leq\left|G\left(x, y_{1}, h_{1}\left(x_{1}\right)\right)-G\left(x, y_{1}, h_{2}\left(x_{2}\right)\right)\right|-b \\
& \leq r M_{p_{B}, J}(h(t), k(t))-b .
\end{aligned}
$$

From (3.14) and (3.15), we have

$$
\left|K h_{1}(x)-K h_{2}(x)\right|+b \leq r M_{p_{B}, J}(h(t), k(t)) .
$$

As the above inequality is true for any $x \in W$ and $\lambda>0$ is taken arbitrarily, so from (3.13) we obtain

$$
\omega(r) p_{B}\left(K h_{1}, J h_{2}\right) \leq p_{B}\left(J h_{1}, I h_{2}\right)
$$

implies

$$
p_{B}\left(K h_{1}, K h_{2}\right) \leq r M_{p_{B},}(h(t), k(t)) .
$$

Therefore, by Corollary B, the pair $(K, J)$ has a common fixed point $h^{*}$, that is, $h^{*}(x)$ is a unique, bounded and common solution of (3.3) and (3.4).

\section{Competing interests}

The authors declare that they have no competing interests.

\section{Authors' contributions}

All authors read and approved the final manuscript.

\section{Author details}

'Department of Mathematics, Lahore University of Management Sciences, Lahore, 54792, Pakistan. ${ }^{2}$ Department of Mathematics, University of Management and Technology, C-II, Johar Town, Lahore, Pakistan.

\section{Acknowledgements}

The authors would like to thank the editor and anonymous reviewers for their useful comments that helped to improve the presentation of this paper.

Received: 9 October 2012 Accepted: 12 January 2013 Published: 31 January 2013

\section{References}

1. Baskaran, R, Subrahmanyam, PV: A note on the solution of a class of functional equations. Appl. Anal. 22(3-4), 235-241 (1986)

2. Markin, J: A fixed point theorem for set valued mappings. Bull. Am. Math. Soc. 74, 639-640 (1968)

3. Nadler, SB: Multi-valued contraction mappings. Pac. J. Math. 30, 475-488 (1969)

4. Ćirić, L: Fixed points for generalized multi-valued contractions. Mat. Vesn. 9, 265-272 (1972)

5. Ćirić, L: Multi-valued nonlinear contraction mappings. Nonlinear Anal. 71, 2716-2723 (2009)

6. Covitz, H, Nadler, SB: Multi-valued contraction mappings in generalized metric spaces. Isr. J. Math. 8, 5-11 (1970)

7. Daffer, PZ, Kaneko, H: Fixed points of generalized contractive multi-valued mappings. J. Math. Anal. Appl. 192 655-666 (1995)

8. Reich, S: Fixed points of contractive functions. Boll. Unione Mat. Ital. 5, 26-42 (1972)

9. Semenov, PV: Fixed points of multi-valued contractions. Funct. Anal. Appl. 36, 159-161 (2002)

10. Naimpally, SA, Singh, SL, Whitfield, JHM: Coincidence theorems for hybrid contractions. Math. Nachr. 127, 177-180 (1986)

11. Singh, SL, Mishra, SN: Nonlinear hybrid contractions. J. Natur. Phys. Sci. 5/8, 191-206 (1991/1994)

12. Singh, SL, Mishra, SN: On a Ljubomir Ćirić fixed point theorem for nonexpansive type maps with applications. Indian J. Pure Appl. Math. 33, 531-542 (2002)

13. Singh, SL, Mishra, SN: Coincidence theorems for certain classes of hybrid contractions. Fixed Point Theory Appl. 2010 Article ID 898109 (2010) 
14. Singh, SL, Mishra, SN: Remarks on recent fixed point theorems. Fixed Point Theory Appl. (2010). doi:10.1155/2010/452905

15. Suzuki, T: A generalized Banach contraction principle that characterizes metric completeness. Proc. Am. Math. Soc. 136, 1861-1869 (2008)

16. Ali, B, Abbas, M: Suzuki type fixed point theorem for fuzzy mappings in ordered metric spaces. Fixed Point Theory Appl. 2013, Article ID 9 (2013)

17. Ćirić, L, Abbas, M, Rajović, M, Ali, B: Suzuki type fixed point theorems for generalized multi-valued mappings on a set endowed with two b-metrics. Appl. Math. Comput. 219, 1712-1723 (2012)

18. Dhompongsa, S, Yingtaweesittikul, H: Fixed points for multi-valued mappings and the metric completeness. Fixed Point Theory Appl. 2009, Article ID 972395 (2009)

19. Kikkawa, M, Suzuki, T: Three fixed point theorems for generalized contractions with constants in complete metric spaces. Nonlinear Anal. 69, 2942-2949 (2008)

20. Kikkawa, M, Suzuki, T: Some similarity between contractions and Kannan mappings. Fixed Point Theory Appl. 2008, Article ID 649749 (2008)

21. Moț, G, Petruşel, A: Fixed point theory for a new type of contractive multi-valued operators. Nonlinear Anal. 70 3371-3377 (2009)

22. Matthews, SG: Partial metric topology. In: Proc. 8th Summer Conference on General Topology Appl. Ann. New York Acad. Sci., vol. 728, pp. 183-197 (1994)

23. Bari, CD, Vetro, P: Fixed points for weak $\varphi$-contractions on partial metric spaces. Int. J. Eng. Contemp. Math. Sci. 1, 5-13 (2011)

24. Paesano, D, Vetro, P: Suzuki's type characterizations of completeness for partial metric spaces and fixed points for partially ordered metric spaces. Topol. Appl. 159, 911-920 (2012)

25. Ćirić, L, Samet, B, Aydi, H, Vetro, C: Common fixed points of generalized contractions on partial metric spaces and an application. Appl. Math. Comput. 218, 2398-2406 (2011)

26. Heckmann, R: Approximation of metric spaces by partial metric spaces. Appl. Categ. Struct. 7, 71-83 (1999)

27. Romaguera, S: A Kirk type characterization of completeness for partial metric spaces. Fixed Point Theory Appl. 2010, Article ID 493298 (2010)

28. Schellekens, MP: The correspondence between partial metrics and semivaluations. Theor. Comput. Sci. 315, 135-149 (2004)

29. Aydi, H, Abbas, M, Vetro, C: Partial Hausdorff metric and Nadler's fixed point theorem on partial metric spaces. Topol. Appl. 159, 3234-3242 (2012)

30. Zamfirescu, T: Fixed point theorems in metric spaces. Arch. Math. 23, 292-298 (1972)

31. Bellman, R: Methods of Nonlinear Analysis. Vol. Il. Mathematics in Science and Engineering, vol. 61. Academic Press, New York (1973)

32. Bellman, R, Lee, ES: Functional equations in dynamic programming. Aequ. Math. 17, 1-18 (1978)

33. Bhakta, PC, Mitra, S: Some existence theorems for functional equations arising in dynamic programming. J. Math. Anal. Appl. 98, 348-362 (1984)

34. Pathak, HK, Cho, YJ, Kang, SM, Lee, BS: Fixed point theorems for compatible mappings of type $P$ and applications to dynamic programming. Matematiche 50, 15-33 (1995)

35. Altun, I, Simsek, H: Some fixed point theorems on dualistic partial metric spaces. J. Adv. Math. Stud. 1, 1-8 (2008)

36. Altun, I, Sola, F, Simsek, H: Generalized contractions on partial metric spaces. Topol. Appl. 157, 2778-2785 (2010)

37. Abbas, $M$, Nazir, T: Fixed point of generalized weakly contractive mappings in ordered partial metric spaces. Fixed Point Theory Appl. 2012, Article ID 1 (2012)

38. Bukatin, MA, Shorina, SY: Partial metrics and co-continuous valuations. In: Nivat, M et al. (eds.) Foundations of Software Science and Computation Structure. Lecture Notes in Comput. Sci., vol. 1378, pp. 125-139. Springer, Berlin (1998)

39. Romaguera, S, Valero, O: A quantitative computational model for complete partial metric spaces via formal balls. Math. Struct. Comput. Sci. 19, 541-563 (2009)

doi:10.1186/1687-1812-2013-21

Cite this article as: Abbas and Ali: Fixed point of Suzuki-Zamfirescu hybrid contractions in partial metric spaces via partial Hausdorff metric. Fixed Point Theory and Applications 2013 2013:21.

\section{Submit your manuscript to a SpringerOpen ${ }^{\ominus}$ journal and benefit from:}

- Convenient online submission

Rigorous peer review

- Immediate publication on acceptance

- Open access: articles freely available online

- High visibility within the field

- Retaining the copyright to your article

Submit your next manuscript at $>$ springeropen.com 